\title{
DENDROCRONOLOGIA, INCREMENTO E MANEJO FLORESTAL EM FLORESTA DE ARAUCÁRIA-SC
}

\author{
DENDROCHRONOLOGY, INCREMENT AND FOREST MANAGEMENT IN ARAUCARIA FOR- \\ EST, SANTA CATARINA STATE
}

\author{
André Felipe Hess ${ }^{1}$ Pollyni Ricken ${ }^{2}$ Lucas Dalmolin Ciarnoschi ${ }^{2}$
}

\begin{abstract}
RESUMO
O manejo sustentável é de extrema importância para a preservação futura, uso múltiplo, produção rentável e valorização da Floresta com Araucária no sul do Brasil. Assim, com as medidas da largura dos anéis de incremento é possível ajustar o crescimento da espécie no tempo, para obtenção do ciclo de corte, ordenamento da produção e manejo da floresta. Os objetivos desse trabalho foram os de estudar o incremento em diâmetro para araucária, modelar o crescimento em função da dimensão e correlacionar o incremento com índices de competição para promover o manejo sustentável da espécie. Para tanto, a floresta foi inventariada utilizando processo de amostragem sistemático, com 25 parcelas de $400 \mathrm{~m}^{2}$ e coletadas as informações dendrométricas, morfométricas e do incremento com uso da dendrocronologia de 121 árvores distribuídas em três classes de diâmetro. A espécie demonstrou incremento diamétrico variável com a idade e correlação negativa com os índices de competição. A estrutura temporal em diâmetro com base na dinâmica do seu incremento médio anual mostrou um aumento de $74,42 \%$ no número de indivíduos nas classes comerciais para um período de 25 anos. A espécie apresentou um incremento médio em diâmetro de $0,708 \mathrm{~cm} \cdot \mathrm{ano}^{-1} \mathrm{e}$ uma taxa de corte viável de $36,59 \mathrm{~m}^{3}$.ha ${ }^{-1}$ para um ciclo entre cortes de 25 anos.
\end{abstract}

Palavras-chave: anéis de crescimento; manejo florestal; prognose florestal.

\begin{abstract}
Sustainable management is of extreme importance for future preservation, multiple use, profitable production and enhancement of Araucaria Forest in southern Brazil. Thus, with the width measures of the increment rings, it is possible to adjust the growth of species over time, to obtain the cutting cycle, plan the production and manage the species. The objectives of this study were to study the increase in diameter to Araucaria, model the growth on the size and correlate the increase in competition indices to promote the sustainable management of the species. Therefore, the forest was inventoried using systematic sampling process, with 25 plots of $400 \mathrm{~m}^{2}$ and collected the dendrometric information, morphometric and increase with use of dendrochronology of 121 trees distributed in three diameter classes. The species showed diameter increment variable with age and negative correlation with the competition indices. The temporal structure in diameter based on the dynamics of its annual average increment showed an increase of $74.42 \%$ in the number of individuals in commercial classes for a period of 25 years. The species showed an average increment in diameter of $0.708 \mathrm{~cm} . \mathrm{ano}^{-1}$ and a viable cut rate of $36.59 \mathrm{~m}^{3} \cdot \mathrm{ha}^{-1}$ for one cycle between cuts of 25 years.
\end{abstract}

Keywords: growth rings; forest management; diametric structure.

1 Engenheiro Florestal, Dr., Professor do Departamento de Engenharia Florestal, Universidade do Estado de Santa Catarina, Av. Luiz de Camões, 2090, Conta Dinheiro, CEP 88520-000, Lages (SC), Brasil. hessandre@yahoo. com.br

2 Engenheiros Florestais, MSc., Doutorandos do Programa de Pós-Graduação em Engenharia Florestal da Universidade Federal do Paraná, Av. Prefeito Lothário Meissner, 3400, Jardim Botânico, CEP 80210-170, Curitiba (PR), Brasil.pollyni7@hotmail.com / ciarnoschi@hotmail.com

Recebido para publicação em 30/10/2014 e aceito em 6/02/2018

Ci. Fl., v. 28, n. 4, out. - dez., 2018 


\section{INTRODUÇ̃̃O}

Araucária, pinheiro brasileiro, Araucaria angustifolia (Bertol.) Kuntze é uma árvore dioica que domina a Floresta Ombrófila Mista, conhecida como Floresta de Araucária (VELOSO; RANGEL FILHO; LIMA, 1991). Ela pertence à família Araucariaceae, coníferas primitivas do hemisfério Sul e originadas no período Triássico (CATTANEO et al., 2013). A araucária é uma espécie pioneira, intolerante à sombra que domina o dossel superior (HUECK, 1972). Originalmente, a Floresta de Araucária ocupava $177.600 \mathrm{Km}^{2}$ no Brasil e $2.100 \mathrm{Km}^{2}$ na Argentina (LEITE; KLEIN, 1990; GIRAUDO et al., 2003), em uma extensão entre $19^{\circ} 15^{\prime} \mathrm{S}-31^{\circ} 31^{\prime} \mathrm{S} \mathrm{S}$ e $41^{\circ} 31^{\prime} \mathrm{O}-54^{\circ} 30^{\prime} \mathrm{O}$ e altitude variando de 200 a $1.800 \mathrm{~m}$ (CARVALHO, 1994).

Devido à exploração sem conhecimento dos elementos necessários ao manejo sustentável, a legislação proibiu a exploração da espécie, como medida de conservar e preservar as áreas remanescentes com floresta de araucária. Contudo, a não intervenção não garante a conservação das florestas, pois ocorre estagnação do crescimento, aumento do ciclo de rotação final, o desinteresse social em áreas de florestas e baixo índice de regeneração natural (BECKERT; ROSOT; ROSOT, 2014). De acordo com Sullivan e Sullivan (2016), não favorecendo o estabelecimento de atributos estruturais de florestas primárias.

Assim, o problema está em conhecer informações sobre incremento, o crescimento no tempo, qual ciclo de corte entre intervenções, a produtividade, taxa de regeneração, entre outras, para que se estabeleça o manejo sustentável da espécie visando ao uso múltiplo e perpetuidade dos seus remanescentes (MATTOS et al., 2007). Segundo Souza et al. (1993), o manejo das florestas nativas passa, obrigatoriamente, pelo conhecimento dos processos de dinâmica de seu crescimento, como também é necessário saber como e quanto as intervenções silviculturais afetam o crescimento das árvores da floresta manejada.

O estudo do incremento e dinâmica do crescimento envolve a análise de anéis de crescimento de espécies comerciais (dendrocronologia), pois fornecem informações sobre a idade da árvore, da dinâmica florestal, dos impactos e associações do crescimento com o clima (SPEER, 2010).

Contribuir com o estabelecimento do manejo racional da floresta exige dados confiáveis de crescimento (CUNHA; FINGER, 2013). Spiecker (2002) comenta que o manejo florestal tem consequências duradouras, as informações atualizadas das condições atuais e futuras da floresta são especialmente importantes, sendo necessária uma previsão mais confiável sobre o desenvolvimento futuro dos recursos florestais e melhor compreensão das mudanças ecológicas, econômicas e sociais.

Segundo a Food and Agriculture Organization of the United Nations (2007), em todo o mundo, a desaparição de florestas primárias, naturais ou não manejadas é de grande preocupação. Intervenções florestais são importantes já que contribuem para a aceleração da sucessão florestal e ajudam a floresta a desenvolver características estruturais finais já em bosques mais jovens (BAUHUS; PUETTMANN; MESSIER, 2009). Florestas não manejadas de araucária apresentaram produção e crescimento estável, ou diminuição ao longo do tempo, baixo ou inexistente recrutamento de novos indivíduos, baixas taxas de incremento, competição, limitada disponibilidade de recursos e superioridade das taxas de mortalidade, o que pode indicar uma tendência ao desaparecimento da espécie se não se realizar nenhuma intervenção silvicultural (BECKERT; ROSOT; ROSOT, 2014).

Uma forma de conservar os remanescentes da Floresta com Araucária é estimular o seu manejo em bases sustentáveis. Até o momento, pouca atenção foi direcionada à obtenção, interpretação e aplicação dos elementos de monitoramento da produtividade primária, da dinâmica de populações, de crescimento, das taxas de regeneração natural e mortalidade (MATTOS et al., 2007).

Neste sentido, o objetivo do estudo foi o de estudar a dinâmica de crescimento de araucária pela análise e medição do incremento médio anual em diâmetro, modelar funções e calcular a taxa de corte sustentada, correlacionar o incremento com índices de competição e realizar análise futura da distribuição diamétrica de classes comerciais com base no seu incremento médio, sendo informações necessárias para auxiliar o manejo florestal e conservação da espécie. 


\section{MATERIAIS E MÉTODOS}

\section{Sítio de estudo}

Uma floresta nativa, com 84 ha, de Araucaria angustifolia foi selecionada como sítio de estudo na região Sul de Santa Catarina, no município de Lages (Figura 1), em altitude de $1.200 \mathrm{~m}$ e coordenadas geográficas central da área de $27^{\circ} 49^{\prime} \mathrm{S}$ e $50^{\circ} 06^{\prime} \mathrm{O}$. A região é classificada segundo Köppen como clima Cfb, temperado constantemente úmido, com verão sem estação seca. A temperatura média anual é de $15,2^{\circ} \mathrm{C}$, a precipitação pluviométrica média anual é de $1.684 \mathrm{~mm}$ e, a umidade relativa do ar varia de $80 \%$ a $83 \%$ (ALVARES et al., 2013).

Os solos da região são associações de solos litólicos distróficos e eutróficos, com horizonte A proeminentemente moderado, textura argilosa, fase rochosa, relevo fortemente ondulado e montanhoso. Ainda, há a ocorrência de solos da classificação cambissolo álico ( $\mathrm{Tb} \mathrm{A}$ ) proeminente, textura muito argilosa, relevo forte ondulado e ondulado com afloramentos rochosos, relevo forte ondulado e montanhoso (EMPRESA DE PESQUISA AGROPECUÁRIA E EXTENSÃO RURAL DE SANTA CATARINA, 2002).

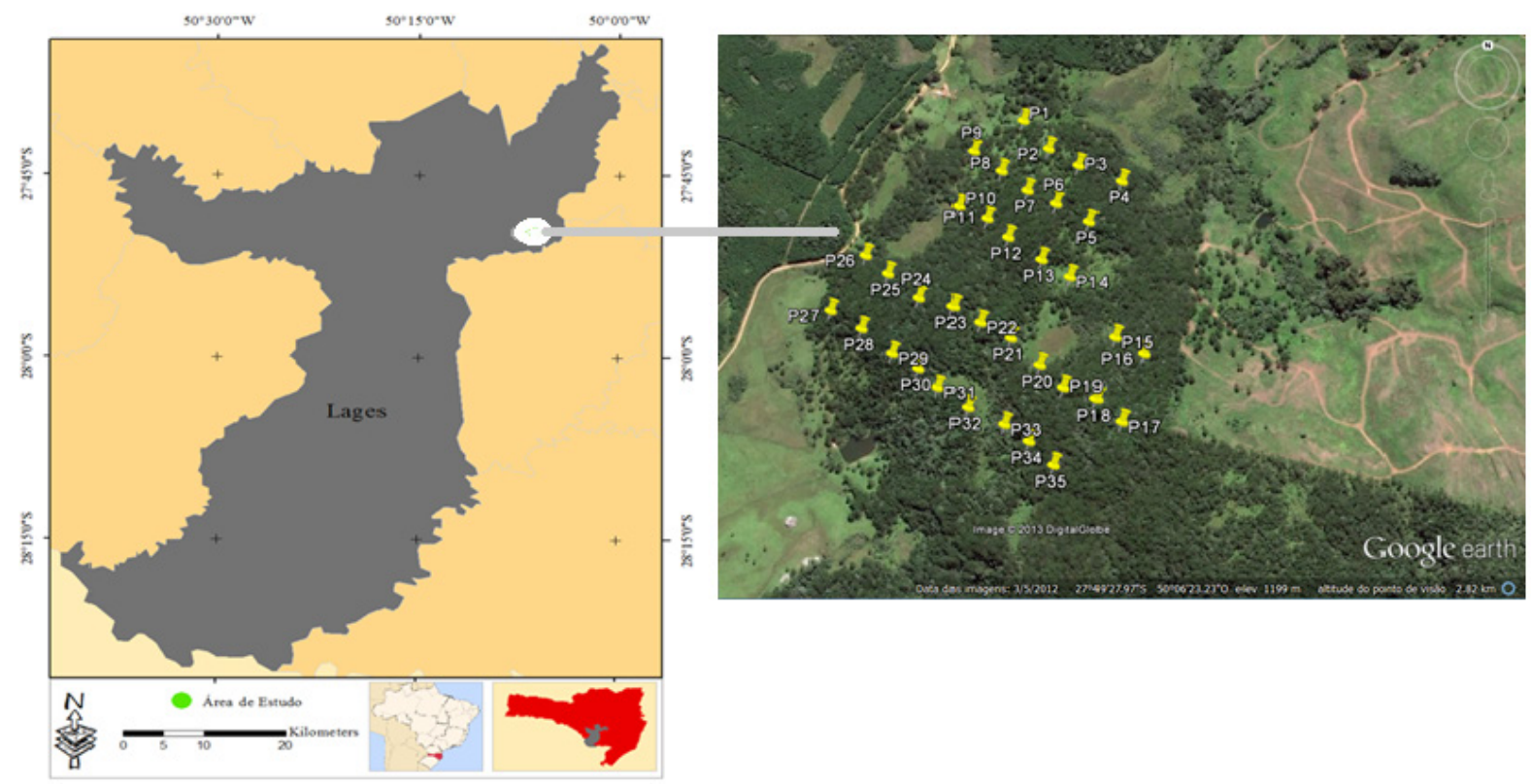

FIGURA 1: Localização da área de estudo no município de Lages e das 34 parcelas amostrais em Floresta de Araucária, Santa Catarina.

FIGURE 1: Study area located in the municipality of Lages and the 34 sample plots in Araucaria Forest, Santa Catarina state.

\section{Coleta de dados}

\section{Variáveis dedrométricas e morfométricas}

No sítio de estudo foi realizado inventário sistemático, em faixas, com equidistância de $100 \mathrm{~m}$. Em cada faixa foram alocadas parcelas de $20 \times 20 \mathrm{~m}\left(400 \mathrm{~m}^{2}\right)$, distantes $50 \mathrm{~m}$ entre si. Em cada parcela foram coletados dados dendrométricos e morfométricos de todos os indivíduos a partir de $10 \mathrm{~cm}$ de diâmetro a altura do peito (dap). Na parcela foram georreferenciados os indivíduos dominantes e suas competidoras, com uso de GPS (Garmin II). A intensidade amostral da área foi obtida com a equação empregada por Sanquetta et al. (2009), considerando $10 \%$ de erro de amostragem.

Foram medidas as variáveis dendrométricas (dap) e quatro raios de copa nas direções cardinais (Norte, Sul, Leste e Oeste) com bússola. Com as quatro projeções dos raios de copa obtiveram-se, com o conjun- 
to de equações abaixo, as informações do raio médio de copa, do diâmetro de copa; da área de projeção de copas e o número potencial de árvores, conforme Burger (1939), Assmann (1961) e Durlo e Denardi (1998):

$$
\overline{\mathrm{rc}}=\sum_{\mathrm{i}=1}^{\mathrm{n}=4} \frac{\mathrm{rc}}{4},
$$

Em que: $\overline{r c}$ : raio médio de copa das quatro medidas em $\mathrm{m}$.

Por multiplicação deste, o diâmetro de copa:

$\mathrm{Dc}=2 * \overline{\mathrm{rc}}$,

Em que: Dc : diâmetro de copa em m; $\overline{r c}$ : raio médio de copa em $\mathrm{m}$.

A área de projeção de copa:

$$
\mathrm{Ac}=\pi * \overline{\mathrm{rc}}^{2}
$$

Em que: Ac: área de projeção de copa em $\mathrm{m}^{2} ; \overline{r c}^{2}$ : raio de copa médio ao quadrado em (m).

Com os dados da foi obtido o Número Potencial de árvores para cada classe diamétrica da floresta:

$$
\mathrm{NP}=\frac{10.000}{\mathrm{Ac}}
$$

Em que: NP: número potencial de árvores; $\mathrm{Ac}=$ área de projeção da copa em $\left(\mathrm{m}^{2}\right)$.

Para compreensão da dinâmica de crescimento e da relação forma e dimensão compilaram-se gráficos para a variável da área de copa (eq. 3) com os dados de incremento médio anual em diâmetro de árvores na floresta e a distância entre árvores, visando identificar a necessidade de espaço à medida que ocorre o desenvolvimento das árvores na floresta e planejar a necessidade de intervenções silviculturais.

\section{Dendrocronologia, incremento e manejo sustentável de araucária}

Para obtenção do incremento anual em diâmetro foram retirados rolos de incremento utilizando trado de Presler com $30 \mathrm{~cm}$ de comprimento e $5,15 \mathrm{~mm}$ de diâmetro de núcleo, perpendicularmente ao dap (ASSMANN, 1970), de 121 árvores divididas nos estratos dominante, codominante e dominadas. Os rolos de incremento foram secos em temperatura ambiente, colados em porta baguetas, lixados e marcados os anéis. Com auxílio de um microscópio estereoscópio foram medidos os incrementos em diâmetro em mesa de mensuração com precisão de $0,01 \mathrm{~mm}$, utilizando o Lintab 6. Os dados foram analisados no Programa Time Series Analysis Program - TSAP -Win da empresa Rinntech ${ }^{\circ}$.

A datação dos anéis foi realizada em planilha eletrônica, filtrando-se e excluindo-se falsos anéis para cada árvore de forma individual. De acordo com os anéis de crescimento para o conjunto das árvores, foi possível medir informação do incremento anual de idades entre 13 a 62 anos ao nível de dap, não representando a idade real da árvore, já que, até a altura do dap, há perda de anéis de crescimento, mas viabiliza a obtenção do incremento anual, idade aproximada e efetivação de modelos de crescimento.

Os indivíduos foram separados em três classes de diâmetro. A classe 1 (C1) dominadas, foi formada com indivíduos que apresentaram dap de $10 \mathrm{~cm}$ a $29,9 \mathrm{~cm}$, a classe 2, codominantes (C2) com indivíduos entre $30 \mathrm{~cm}$ e $49,9 \mathrm{~cm}$ e a classe 3 , dominantes (C3) com aqueles acima de $50 \mathrm{~cm}$. Os valores de incremento médio anual em diâmetro entre as classes foram avaliados empregando-se teste não paramétrico de Kruskal-Wallis, pois os dados não apresentaram normalidade para o teste de Kolmogorov-Smirnov, valor de $0,0818(P<0,439)$, para um nível de confiança de $95 \%$, com objetivo de verificar se existe ou não diferenças no crescimento entre as classes.

Com os dados do incremento em diâmetro a cada ano e subtraindo o incremento anual do diâmetro atual, foi possível regredir o dap no tempo e ajustar modelo de altura em função do dap para cada ano, com uso da técnica de regressão dos modelos lineares generalizados (MLG). A acurácia do modelo foi obtida pelo valor do desvio $(22,1)$ e critério de Akaike $(2093,9)$, sendo que quanto menor o valor do critério, melhor o ajuste.

$$
\mathrm{h}=7,4373+0,2077 * \text { dap }
$$

Em que: h: altura em m; dap: diâmetro a altura do peito em $\mathrm{cm}$.

Com os dados do dap e altura para cada ano foi calculado o volume utilizando a equação 6 , conforme 
Hess et al. (2010), sendo possível obter o volume anual com a relação entre o diâmetro e altura a cada ano:

$$
\mathrm{V}=0,0071+0,00005 * \mathrm{dap}^{2}+0,00003 * \mathrm{dap}^{2} * \mathrm{~h}+0,00004 * \text { dap } * \mathrm{~h}^{2}-0,0006 * \mathrm{~h}^{2}
$$

Em que: V: volume em $\mathrm{m}^{3}$; dap: diâmetro a altura do peito em $\mathrm{cm}$; h: altura em $\mathrm{m}$.

A acurácia do modelo apresentou valores de $\mathrm{R}^{2}$ aj. de 0,99 , erro padrão da estimativa (Syx) de 0,0254 , coeficiente de variação de 4,16 \% e valor F de 26277,8.

Após obtenção do volume em cada ano calculou-se o incremento periódico percentual em volume para um período de 10 anos com a equação 7 :

$$
\operatorname{IPA}_{\mathrm{v}} \%=\left(\frac{\mathrm{IP}}{\mathrm{V}_{\mathrm{i}}}\right) * \frac{100}{\mathrm{n}}
$$

Em que: IPA $\%$ : incremento periódico anual percentual em volume; $\mathrm{IP}_{\mathrm{V}}$ : incremento periódico em volume em $\mathrm{m}^{3}$, obtido com a expressão $\left(\mathrm{v}_{\mathrm{i}}-\mathrm{v}_{10}\right)$, em que $\mathrm{v}_{\mathrm{i}}$ : volume no final do período e $\mathrm{v}_{10}$ : volume no início do período; $\mathrm{v}_{\mathrm{i}}$ : volume $\mathrm{em}^{3}$ no final do período, $\mathrm{n}$ : número de anos do período.

A intensidade de corte foi calculada para um ciclo de corte de 25 anos. A escolha do ciclo de corte teve como base a taxa de incremento médio anual em diâmetro, bem como o trabalho de Hess et al. (2010) e a ponderação para intervenções silviculturais com cautela, para que a floresta consiga desenvolver atributos de florestas primárias, com árvores de velho crescimento (BAUHUS; PUETTMANN; MESSIER, 2009).

$$
\mathrm{IC}=\left[1-\left(\frac{1}{1,0 \mathrm{IPA} \mathrm{A}_{V} \mathrm{CC}}\right)\right] * 100
$$

Em que: IC: intensidade de corte em percentagem do volume; IPA \%: incremento periódico anual percentual em volume; cc: ciclo de corte para um período de 25 anos.

A taxa de corte foi obtida com a seguinte equação:

$\mathrm{TC}=\mathrm{Vr} * \frac{\mathrm{IC}}{100}$

Em que: TC: taxa de corte em volume $\left(\mathrm{m}^{3}\right)$ para o ciclo de corte; Vr: volume real disponível na floresta $\mathrm{em}^{3}$.

Com os dados do incremento anual em diâmetro e do dap de cada árvore de cada classe, ajustou-se a equação 10 para cada ano medido no rolo de incremento, a relação entre dimensão-crescimento para as árvores amostras de araucária foi demonstrada de forma linear, objetivando demonstrar a interdependência do aumento em dimensão (dap) com a taxa de incremento médio anual em diâmetro, conforme Pretzsch e Dieler (2011).

$$
\mathrm{id}=\mathrm{a}_{0}+\mathrm{a}_{1} * \text { dap }+\varepsilon
$$

Em que: id: incremento médio anual em diâmetro em $\mathrm{cm}^{\text {ano }}{ }^{-1}$; dap: diâmetro a altura do peito em $\mathrm{cm}$; $\mathrm{a}_{\mathrm{s}}$ : coeficientes do modelo; $\varepsilon$ : erro do modelo.

Visando mostrar o efeito do aumento em dimensão rearranjou-se a eq. 10 e utilizou-se o diâmetro médio quadrático da floresta $(\mathrm{dg})$ como variável independente.

$\mathrm{a}_{1}=\mathrm{a}_{0}+\mathrm{id} * \mathrm{dg}+\varepsilon$

Em que: $a_{1}$ : coeficiente angular do modelo; $\mathrm{a}_{0}$ : intercepto; id: incremento médio anual em diâmetro em cm.ano ${ }^{-1}$; dg: diâmetro médio quadrático calculado de $28,1 \mathrm{~cm}$ para a floresta; $\varepsilon$ : erro do modelo.

Usando a eq. 11 a inclinação $a_{1}$ da linha de regressão foi calculada e o respectivo intercepto $a_{0}$ foi derivado por rearranjo da equação:

$$
\mathrm{a}_{0}=\mathrm{id}-\mathrm{a}_{1} * \mathrm{dg}
$$

Aumentando-se o dg da floresta para $35 \mathrm{~cm}$ e substituindo os valores de id - dg foi possível verificar a distribuição relativa do crescimento e incremento das árvores para as diferentes classes de diâmetro, o que é refletido pela diferença de inclinação das linhas de regressão e que podem ser demonstradas graficamente (PRETZSCH; DIELER, 2011).

Assim, na criação deste modelo e incluindo o dg como variável independente, verifica-se o fato bem conhecido de que a inclinação (coeficiente $\mathrm{a}_{1}$ ) para a relação crescimento-dimensão diminui com aumento médio em dimensão das árvores na floresta (PRODAN, 1965).

Com os dados do incremento médio anual em diâmetro foram construídas as trajetórias do cresci- 
mento acumulado em diâmetro, possibilitando identificar o padrão de crescimento, ritmo de crescimento, ponto de inflexão ou estagnação do crescimento.

Os dados do incremento periódico anual em diâmetro para os últimos dez anos foram correlacionados com índices de competição utilizando análise de correlação de Person. Foram utilizados índices de competição dependentes da distância (Tabela 1). Em cada parcela foi determinada a árvore dominante como árvore-objetivo e competidoras as que estavam sob influência da copa da árvore-objetivo.

TABELA 1: Índices de competição calculados para avaliar a relação com o incremento médio em diâmetro para Araucaria angustifolia, Lages - SC.

TABLE 1: Competition indices calculated to assess the relationship with the average increase in the diameter for Araucaria angustifolia, Lages, SC state.

\begin{tabular}{|c|c|}
\hline Índices & Fórmula \\
\hline IC1 & $\sum_{j=1}^{n}\left\lfloor\frac{d a p_{j}}{\operatorname{dap}_{1}} * \operatorname{arctang} \frac{\operatorname{dap}_{j}}{\text { Dist }_{i j}}\right\rfloor$ \\
\hline IC2 & $\sum_{\mathrm{j}=1}^{\mathrm{n}}\left[\frac{\mathrm{h}_{\mathrm{j}}}{\mathrm{h}_{\mathrm{i}}} * \operatorname{arctang} \frac{\mathrm{h}_{\mathrm{j}}}{\text { Dist }_{\mathrm{ij}}}\right]$ \\
\hline IC3 & $\sum_{\mathrm{j}=1}^{\mathrm{n}} \operatorname{arctng}\left(\frac{\mathrm{h}_{\mathrm{j}}}{\text { Dist }_{\mathrm{ij}}}\right)$ \\
\hline IC4 & $\sum_{\mathrm{j}=1}^{\mathrm{N}} \operatorname{arctang}\left(\frac{\text { dap }_{\mathrm{j}}}{\text { Dist }_{\mathrm{ij}}}\right)$ \\
\hline IC5 & $\sum_{j=1}^{N}\left[\frac{h_{j}}{\left(h_{i} * \text { Dist }_{i j}\right)}\right]$ \\
\hline IC6 & $\sum_{j=1}^{n}\left[\frac{d_{j}}{\left(d_{i} * \text { Dist }_{i j}\right)}\right]$ \\
\hline IC7 & $\sum_{j=1}^{n}\left[\frac{\text { dap }_{j}}{\left(\operatorname{dap}_{i} * \text { Dist }_{i j}^{0.5}\right)}\right]$ \\
\hline
\end{tabular}

Em que: dap: diâmetro a altura do peito; h: altura em m; Dist: distância horizontal; n: todas as árvores vizinhas consideradas competidoras, i: i-ésima árvore objetivo; j: j-ésima árvore competidora.

Com base no incremento médio anual em diâmetro, foi realizada análise futura da estrutura de distribuição diamétrica de classes comerciais para um período de 25 anos. O objetivo dessa projeção foi identificar e ilustrar a quantidade de indivíduos a partir da classe de $40 \mathrm{~cm}$, pois é o diâmetro mínimo de corte estipulado no manejo florestal da espécie.

Os dados de incremento médio anual em diâmetro foram submetidos aos testes de normalidade (Shapiro-Wilk W) e homogeneidade da variância (teste de White). Todas as análises foram processadas no programa SAS 9.3 (Statistc Analisys System). O teste de White acusou um valor de $\chi^{2}$ igual a 5,67 não significativo $(\mathrm{p}=0,058)$, portanto, os dados de incremento não apresentam heterogeneidade da variância. $\mathrm{O}$ teste de Shapiro-Wilk apresentou valor de 0,97 não significativo na probabilidade de 0,1085 , deste modo, os dados de incremento atendem à condição de normalidade com valor de assimetria de 1,28 e curtose de -0,57. 


\section{RESULTADOS E DISCUSSÃO}

\section{Dendrometria e morfometria}

Os cálculos para suficiência amostral apontaram a necessidade de 25 parcelas, demonstrando que com o total amostrado, 35 parcelas, cumpre-se o quesito de suficiência amostral. A frequência total das classes de diâmetro demonstrou uma estrutura diamétrica na forma de J-invertido (Figura 2). A amplitude diamétrica variou de 10 a $87,6 \mathrm{~cm}$, o diâmetro médio da florestal foi de $26,8 \mathrm{~cm}$, o diâmetro médio quadrático de $28,1 \mathrm{~cm}$, a altura média de $12,9 \mathrm{~m}$ e a área basal de $18,8 \mathrm{~m}^{2}$.ha-1. O povoamento apresentou um total de 303 árvores.ha ${ }^{-1}$, das quais 8,25 \% são dominantes, 26,89\% competidoras e 64,86 \% demais indivíduos.

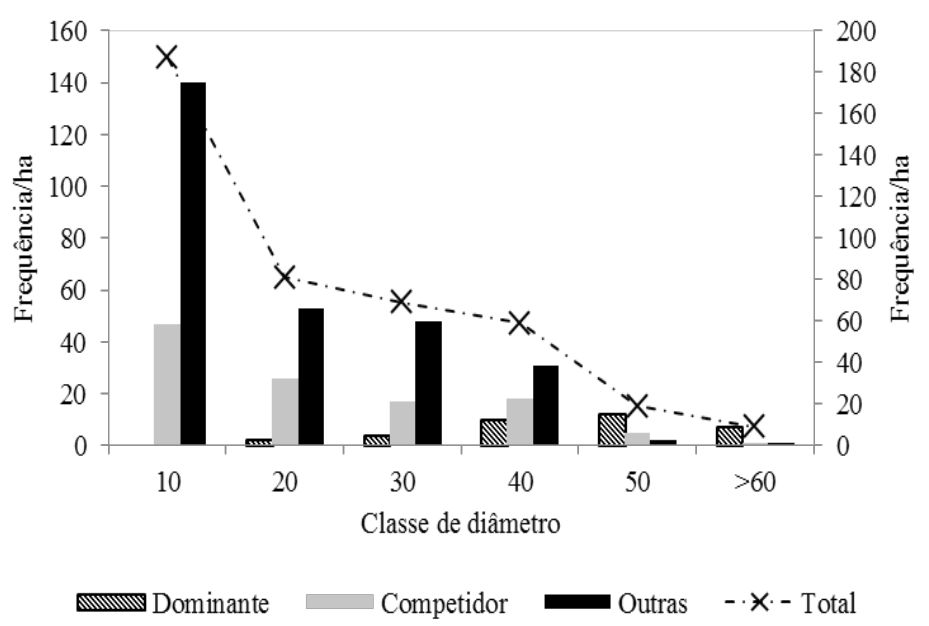

FIGURA 2: Número de árvores por classe diamétrica em floresta nativa de Araucaria angustifolia, Lages - SC. FIGURE 2: Number of trees per diameter class in native forest of Araucaria angustifolia, Lages, SC state.

Utilizando os valores das variáveis morfométricas foi possível obter o número potencial de árvores com base na área de copa para cada classe de diâmetro (Tabela 2).

TABELA 2: Ocupação do espaço e número potencial de árvores em função da classe diamétrica, diâmetro e área da copa.

TABLE 2: Morphometric data for calculation of space occupation and decreasing density with increasing growth and size of trees.

\begin{tabular}{cccc}
\hline & & & Número Potencial \\
Classe de diâmetro & Diâmetro de copa $(\mathrm{m})$ & Área de copa $\left(\mathrm{m}^{2}\right)$ & \\
& & & $\left(\right.$ árvores.ha $\left.{ }^{-1}\right)$ \\
\hline 10 & 3,54 & 9,83 & 1017 \\
15 & 4,83 & 18,3 & 547 \\
20 & 6,55 & 33,7 & 297 \\
25 & 7,43 & 43,32 & 231 \\
30 & 7,87 & 48,63 & 206 \\
35 & 8,83 & 61,17 & 163 \\
40 & 9,38 & 69,12 & 145 \\
45 & 11,7 & 107,44 & 93 \\
50 & 13,13 & 135,47 & 74 \\
$>50$ & 16,7 & 210,54 & 47 \\
\hline
\end{tabular}


Os resultados indicam que o espaçamento médio de crescimento das árvores é inversamente proporcional ao número de árvores e diretamente proporcional ao diâmetro médio (ASSMANN, 1970). Assim, existe correlação entre a variável área de copa, distância entre árvores (espaço de crescimento) e incremento médio em diâmetro (Figura 3), indicando que, para atingir maiores taxas de incremento, maior deve ser a distância entre as árvores e maior a área de copa.

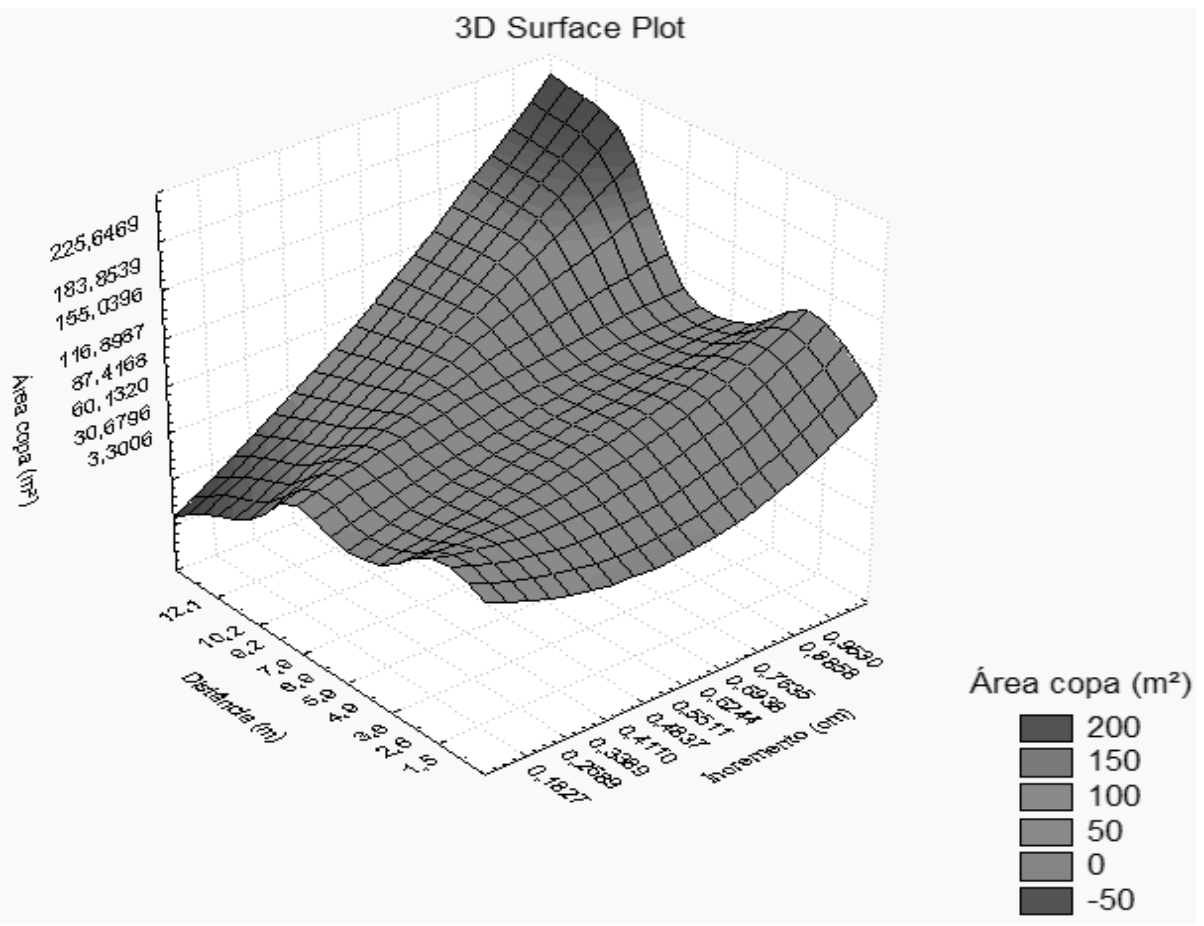

FIGURA 3: Gráfico de superfície demonstrando a inter-relação entre área de copa - incremento médio - distância, das condições atuais das árvores para floresta de Araucaria angustifolia, Lages - SC.

FIGURE 3: Surface graph showing the interrelationship between the crown area - average increment - distances, from the current conditions of the trees to Araucaria angustifolia forest, Lages, SC state.

A taxa de incremento é visivelmente maior em copas que atingiram plena luz solar e decresce à medida que a área de copa diminui, padrão que ocorre devido à variabilidade genética, dos fatores ambientais e recursos para o crescimento, competição e não intervenção de manejo na floresta (SANTOS et al., 2015). Segundo os mesmos autores, a aplicação do método do número de árvores para um espaço disponível indica o período de tempo do crescimento das árvores na floresta em que o espaço individual começa a apresentar comprometimento e o início da competição. A necessidade de espaço pode ser avaliada tanto com o aumento da idade, do dap e variáveis individuais de forma das árvores na estrutura da floresta.

A área de projeção de copa permite que se conheça o espaço ocupado por uma árvore e, quando se dispõe de dados do incremento, que se calcule a produção e a produtividade da mesma (MITSCHERLICH, 1978). Segundo Pretzsch (2009), quando a disponibilidade de recurso é menor no povoamento do que a necessária para o crescimento ideal da árvore, as árvores individuais passam a entrar em concorrência entre si. A visualização da competição correlacionada com as variáveis (Figura 3) permite, assim, compreender a necessidade do arranjo espacial para manter o crescimento e produção.

De forma indireta, os resultados expressam a capacidade fotossintética (produtividade) da árvore e auxiliam em intervenções silviculturais. Assim, quanto menor a expansão lateral da copa, maior a influência das árvores vizinhas (competição), menor a área de copa e menor a taxa de crescimento. Como salientou Assmann (1970), sabe-se que para uma determinada área de projeção de copa, o incremento é maior para árvores com melhor posição sociológica.

Definir a quantidade de árvores para determinada dimensão, diamétrica ou morfométrica, pressupõe conhecer o espaço necessário para que elas se desenvolvam até o período de corte, bem como demonstra a 
dinâmica de desenvolvimento da estrutura diamétrica e temporal do crescimento de árvores na floresta, pois com o crescimento, o espaço ocupado pela árvore (área de projeção de copa) tende a aumentar, conforme Durlo e Denardi (1998).

Estudo e avaliações empregando variáveis morfométricas e relações interdimensionais podem ser utilizados para definir diretrizes de intervenções silviculturais tanto em plantios homogêneos, Tonini e Arco-Verde (2005), Padoin e Finger (2010), como para florestas nativas Durlo e Denardi (1998), Orellana e Koehler (2008), já que são modelos que têm como base as características individuais da espécie em estudo.

\section{Dendrocronologia e manejo florestal}

Os dados obtidos com a medição da largura entre anéis anuais de crescimento permitiram mostrar que o incremento médio anual em diâmetro para o conjunto das árvores foi de $0,708 \mathrm{~cm}$.ano ${ }^{-1} \mathrm{e} \mathrm{com} \mathrm{varia-}$ bilidade de $0,01 \mathrm{~cm} \cdot \mathrm{ano}^{-1}$ a um máximo de $2,37 \mathrm{~cm} \cdot \mathrm{ano}^{-1}$, demonstrando o potencial de crescimento da espécie. A variabilidade do crescimento está relacionada aos fatores do ambiente, recursos do sítio, ontogenia da espécie, existência ou não de intervenção com tratamentos silviculturais, posição da árvore nos estratos e competição.

O teste de médias para os valores de incremento para as três classes de diâmetro, C1, C2 e C3 mostrou existir diferença significativa com valor de $P<0,00000311$ entre as classes $\mathrm{C} 1$ e $\mathrm{C} 2, \mathrm{C} 1$ e $\mathrm{C} 3$, mas não mostrou diferença significativa entre as classes $\mathrm{C} 2-\mathrm{C} 3$ (Tabela 3 ).

TABELA 3: Estatísticas do resultado do teste de médias para avaliar diferenças no valor médio anual de incremento para diferentes classes diamétricas em três estratos em Floresta com Araucária, Lages - SC.

TABLE 3: Statistics of the average test result to evaluate differences in the increase of annual average value for different diameter classes in three strata in Araucaria Forest, Lages, SC state.

\begin{tabular}{cc}
\hline Informação & Valor \\
\hline Kruskal-Wallis qui-quadrado & 25,359 \\
Graus de liberdade & 2 \\
$P$-valor & 0,000 \\
\hline
\end{tabular}

Fatores comparados

Diferença Observada

Diferença Crítica

Diferença

$\begin{array}{llll}\mathrm{C} 1-\mathrm{C} 2 & 48,131 & 23,091 & \text { Sim } \\ \mathrm{C} 1-\mathrm{C} 3 & 29,290 & 22,900 & \text { Sim } \\ \mathrm{C} 2-\mathrm{C} 3 & 18,841 & 23,091 & \text { Não }\end{array}$

A diferença entre as classes $\mathrm{C} 1-\mathrm{C} 2, \mathrm{C} 1$ - C3 é significativa, pois são as árvores que pertencem a estratos diferentes. As árvores da classe 2 e 3 são árvores do dossel superior, isto é, árvores que alcançaram rapidamente o espaço necessário, disponibilidade de recursos e luz, não apresentando diferença significativa, pois mostram características de mesma capacidade de crescimento, uma vez que, em regime de não manejo, são as árvores desses estratos que mais crescem (SOUZA et al., 1993).

Nesse sentido, quando se pensa em formação de uma estrutura futura com árvores de maior dimensão, há a necessidade de intervenções silviculturais para que as classes de menor diâmetro consigam realizar a transição para classes diamétricas de maior dimensão e formar estrutura de floresta primária. Ademais, os componentes estruturais de dimensão e crescimento podem ser manejados, pois, segundo Hosokawa e Solter (1995), para que se produza um uso racional e sobrevivência da floresta, a aplicação de técnicas silviculturais é necessária e planejada quando se conhece o crescimento no tempo da espécie.

A análise do incremento para as três classes de diâmetro demonstrou que, para a classe $\mathrm{C} 1$, o incremento médio foi de $0,54 \mathrm{~cm} \cdot a n o^{-1}$, para $\mathrm{C} 2$, o incremento foi de $0,717 \mathrm{~cm} \cdot \mathrm{ano}^{-1}$ e para $\mathrm{C} 3 \mathrm{de} 0,66 \mathrm{~cm} \cdot \mathrm{ano}^{-1}$. 
O incremento menor nas classes iniciais $(\mathrm{C} 1)$ ocorre em consequência de ser a classe com maior número de indivíduos, característico de floresta não manejada, competição por recursos e espaço, enquanto a redução na C3 deve-se ao fato de serem os indivíduos com idade e diâmetro mais avançado.

Com os dados de incremento em diâmetro anual, foi possível o ajuste da relação hipsométrica e de volume no tempo permitindo determinar o incremento periódico percentual em volume que viabilizou a taxa e intensidade de corte para a estrutura de crescimento atual da floresta (Tabela 4).

TABELA 4: Estatísticas utilizadas para definir o manejo de rendimento sustentado em floresta nativa de Araucaria angustifolia, Lages - SC.

TABLE 4: Statistics used to define the management of sustained yield in native forest of the Araucaria angustifolia, Lages, SC state.

\begin{tabular}{ccccc}
\hline $\mathrm{IPAv} \%$ & $\mathrm{CC}($ ano $)$ & $\mathrm{IC}(\%)$ & $\mathrm{V} / \mathrm{cc}\left(\mathrm{m}^{3}\right)$ & $\mathrm{TC}\left(\mathrm{m}^{3} \cdot \mathrm{ha}^{-1}\right)$ \\
\hline 1,68 & 25 & 33,14 & 110,42 & 36,59 \\
\hline
\end{tabular}

Em que: IPAv\% = incremento periódico percentual em volume; CC: ciclo de corte; IC: intensidade de corte percentual; $\mathrm{V} / \mathrm{c} / \mathrm{c}$ : volume comercial com casca $\mathrm{em} \mathrm{m}^{3}$ para as classes a partir de $40 \mathrm{~cm}$ de dap; TC: taxa de corte $\mathrm{em} \mathrm{m}^{3} \cdot \mathrm{ha}^{-1}$.

Para o povoamento em estudo, obteve-se uma taxa de corte viável de $36,59 \mathrm{~m}^{3}$.ha ${ }^{-1}$, representando para um ciclo de 25 anos uma retirada de 1,464 $\mathrm{m}^{3}$.ha.ano ${ }^{-1}$, ou $122,76 \mathrm{~m}^{3}$ para o total da área, demonstrando a possibilidade de manejo com base no atual valor de incremento médio da floresta. Esse volume representa o valor da sustentabilidade para o ciclo de corte do total de volume comercial $\left(110,42 \mathrm{~m}^{2}\right)$.

\section{Relação crescimento-dimensão}

A análise da relação entre crescimento-dimensão ajustada (equações 10,11,12) mostrou perda proporcional da taxa de incremento em diâmetro com o aumento da dimensão representado pelo coeficiente > 0, (PREZTSCH; DIELER, 2011).

A)

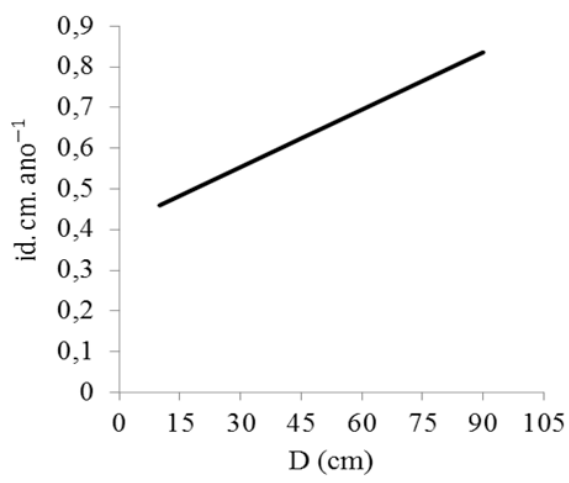

FIGURA 4: Relação entre incremento em diâmetro-diâmetro (id.cm.ano ${ }^{-1} /$ dap) para araucária, Lages - SC.

A) Dados ajustados com os valores atuais para a floresta e, B) Comparação entre dados atuais da floresta e reajuste da linha de regressão do incremento em diâmetro com aumento em dimensão. (id) valor atual da relação incremento-dimensão; (id1): incremento com aumento em dimensão, D: diâmetro a altura do peito em $\mathrm{cm}$.

FIGURE 4: Relationship between diameter increment-diameter (id.cm.ano ${ }^{-1} /$ dap) for Araucaria, Lages, SC state.

A) Adjusted data with the current values for the forest and, B) Comparison of current data of the forest and readjustment of increasing the regression line in diameter with an increase in size. (id) current value of increment-size ratio; (id1): increase with increase in size; D: diameter at breast height in $\mathrm{cm}$. 
Analisando as previsões do incremento com o modelo linear, a participação do crescimento das plantas maiores é elevada na parte superior, intervalo do coeficiente (inclinação da reta), e menor do que o previsto no intervalo inferior (árvores de menores diâmetros). Isso mostra que os resultados do ajuste linear representam estimativas um pouco conservadoras da participação relativa de crescimento e dos recursos para as árvores de maior diâmetro, quando comparado com seus vizinhos menores (PREZTSCH; DIELER, 2011).

A relação entre incremento em diâmetro-diâmetro (id-d) é dependente das condições do sítio, competição, quando todas as outras variáveis que influenciam o crescimento são mantidas constantes. Com o aumento da dimensão ocorre perda proporcional no id (Figura 4B), demonstrado pelo nível inferior da linha de regressão para a relação (id-d), que foi obtido com reajuste da equação 10 , e atribuindo-se um diâmetro médio quadrático (dg) de $35 \mathrm{~cm}$, superior ao atual do povoamento que é de $28,1 \mathrm{~cm}$. Tal ajuste informa a necessidade do manejo de densidade com o aumento da dimensão e diminuição do incremento, o que se verifica nesse povoamento, pois o incremento médio em diâmetro foi de $0,708 \mathrm{~cm}$.ano ${ }^{-1}$, inferior a valores que a araucária pode atingir (se manejada) e encontrados nesse trabalho, superiores a 2,0 cm.ano ${ }^{-1}$ para alguns anos no rolo de incremento.

Para o povoamento em questão, o valor médio de id de $0,708 \mathrm{~cm} \cdot \mathrm{ano}^{-1} \mathrm{e}$ o inferior incremento da classe de 10 a $30 \mathrm{~cm}\left(\mathrm{C} 1 \mathrm{de} 0,54 \mathrm{~cm}^{-a n o^{-1}}\right)$, apesar de ser característico em povoamentos não manejados, indicam a preocupação no desenvolvimento de uma estrutura futura da floresta com características de velho crescimento (old-growth), já que, mesmo com intervenções silviculturais, essas árvores não irão mais inflexionar ou retomar seu crescimento, o que prejudica a produção futura (BAUHUS; PUETTMANN; MESSIER, 2009).

\section{Crescimento acumulado em diâmetro}

$\mathrm{O}$ crescimento acumulado em diâmetro no tempo (classes $\mathrm{C} 1, \mathrm{C} 2$ e $\mathrm{C} 3$ ) demonstrou redução de incremento e estagnação da curva de crescimento, para todas as classes, com poucas árvores apresentando ainda inflexão na curva, tendo atingido a capacidade de suporte. Ainda, na análise das curvas, pode-se concluir que o não manejo (40 anos sem interferência no povoamento) compromete o desenvolvimento individual e da floresta pelas baixas taxas de incremento (decrescente com o tempo), competição, baixa regeneração, rentabilidade futura e expectativa de perpetuidade em número de indivíduos com possibilidade de maior dimensão no futuro (Figura 5).

$\mathrm{Na}$ classe $\mathrm{C} 1$ é possível observar que alguns indivíduos necessitaram de 40 anos para crescer $7 \mathrm{~cm}$ em dap, outros necessitaram de 62 anos para crescer $21 \mathrm{~cm}$, representando um id de apenas $0,3 \mathrm{~cm}^{-a n o^{-1}}$, estando abaixo da capacidade de incremento da espécie, para a qual Mattos et al. (2007) encontraram valor médio de $0,9 \mathrm{~cm}$. ano $^{-1}$. Esse crescimento lento, nas menores classes de diâmetro pode ser resultado da competição que impede seu desenvolvimento e compromete seu crescimento, sendo as intervenções silviculturais uma alternativa para aumento na taxa de crescimento.

Assim, regular a densidade, implica que indivíduos remanescentes alcancem o dossel e dimensão mínima de corte $(40 \mathrm{~cm})$ pelo crescimento rápido na fase jovem. Em outras palavras, indivíduos que têm crescimento lento, podem ao longo do ciclo de corte não atingir dimensão para colheita ou aproveitamento no futuro (ROZENDAAL; GAMBOA; ZUIDEMA, 2010). 

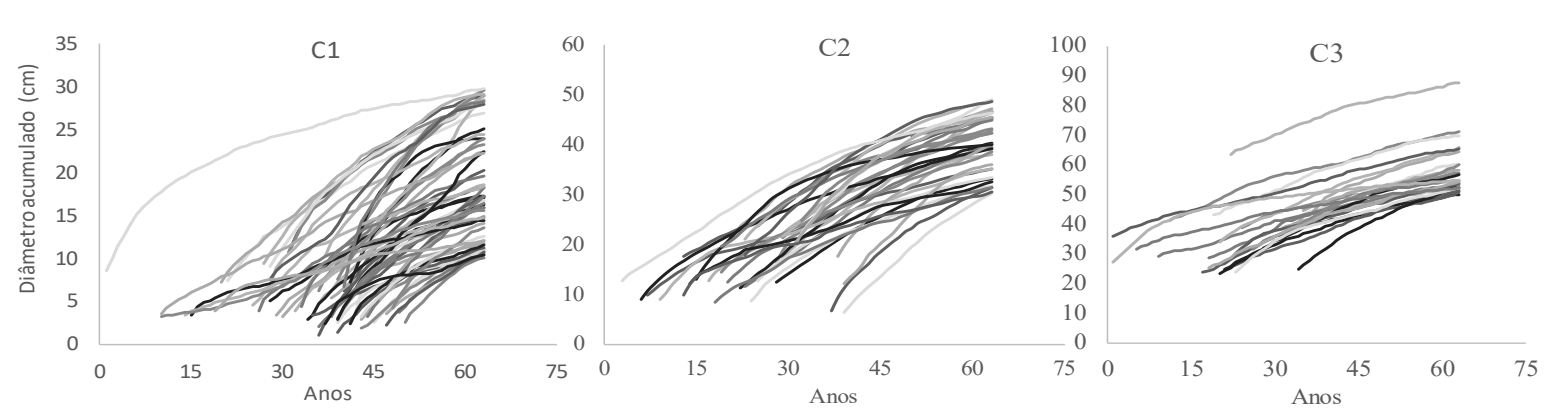

FIGURA 5: Curvas acumuladas do crescimento em diâmetro medido nos rolos de incremento retirados de uma floresta nativa de Araucaria angustifolia, Lages - SC.

FIGURE 5: Accumulated curves of growth measured in diameter increment rolls taken from a native forest of Araucaria angustifolia, Lages, SC state.

A classe $\mathrm{C} 2$ caracteriza as árvores que romperam a competição inicial e que conseguiram alcançar índice de crescimento igual à média do incremento para todas as classes. Na classe $\mathrm{C} 3$, todas as árvores atingiram estagnação de crescimento em diâmetro. A análise das curvas permite concluir que entre 40 a 45 anos as árvores atingiram seu ponto de rotação biológica, ou capacidade máxima de suporte em crescimento. A preocupação maior está na classe $\mathrm{C} 1$, uma vez que um longo período de crescimento e baixas taxas de crescimento significam maior tempo para que as árvores atinjam classes maiores de diâmetro.

Pode-se inferir que a floresta devido à não intervenção de manejo, mostrou que a taxa de incremento das árvores de menor diâmetro é menor que a taxa média de incremento para todos os indivíduos, o que implica a não utilização do crescimento dessas árvores para projeções da produção e rendimento, pois irá gerar subestimativas do crescimento (LAURANCE et al., 2004) devendo-se trabalhar com a taxa média de incremento. Com base no incremento médio em diâmetro, $0,708 \mathrm{~cm} \cdot a^{-1}$, serão necessários 57 anos para produzir uma árvore de $40 \mathrm{~cm}$ de dap, quase duas vezes o tempo de rotação técnica em diâmetro, de 33 anos, conforme Hess, Schneider e Finger (2009).

Além de indicar a condição atual do povoamento, a medição do crescimento também tem como finalidade fornecer informações sobre o futuro desses indivíduos. Essa característica pode ser correlacionada entre o incremento periódico anual e os índices de competição (Tabela 5), que apresentam correlação negativa, indicando que o efeito da competição diminuiu as taxas de incremento.

TABELA 5: Correlação de Pearson para a relação entre incremento periódico anual em diâmetro e índices de competição, para Araucaria angustifolia, Lages - SC.

TABLE 5: Pearson correlation for the relationship between annual periodic increment in diameter and indices competition for Araucaria angustifolia, Lages, SC state.

\begin{tabular}{c|c|c|c|c|c|c|c}
\hline Var & IC1 & IC2 & IC3 & IC4 & IC5 & IC6 & IC7 \\
\hline IPA $_{d}$ & $-0,33$ & $-0,42$ & $-0,3$ & $-0,31$ & $-0,34$ & $-0,28$ & $-0,14$ \\
\hline
\end{tabular}

O efeito negativo da competição no incremento em diâmetro das árvores na floresta mostra que tratamentos silviculturais e o manejo adequado são essenciais para obtenção e manutenção do rendimento da floresta, desenvolvimento de estrutura futura. A intervenção silvicultural pensando em formação de estrutura diamétrica com árvores de maior dimensão e aumento das taxas de incremento é positiva, pois otimiza o espaço de crescimento, a distribuição dos recursos abióticos e bióticos para o crescimento e perpetuidade da floresta com araucária.

A maior correlação foi com o IC2 $(r=-0,42$ e valor $P<0,031)$. A correlação negativa confirma o resultado encontrado na Figura 3, havendo necessidade de abertura de espaço para que a competição diminua e aumente as taxas de incremento, principalmente nas menores classes de diâmetro. 


\section{Dinâmica da estrutura diamétrica}

Com base no id em diâmetro $\left(0,708 \mathrm{~cm}^{-a n o}{ }^{-1}\right)$, semelhante ao id para a classe $\mathrm{C} 2\left(0,71 \mathrm{~cm}\right.$.ano $\left.{ }^{-1}\right)$, árvores de igual ou maior que $40 \mathrm{~cm}$, foi construída uma análise prospectiva para indicar o número de árvores de classes comerciais ( $\geq 40 \mathrm{~cm}$ ) para um período de 25 anos. A Figura 6 mostra o número de indivíduos por classe atual e o cenário futuro, mantendo-se a taxa de incremento na floresta.
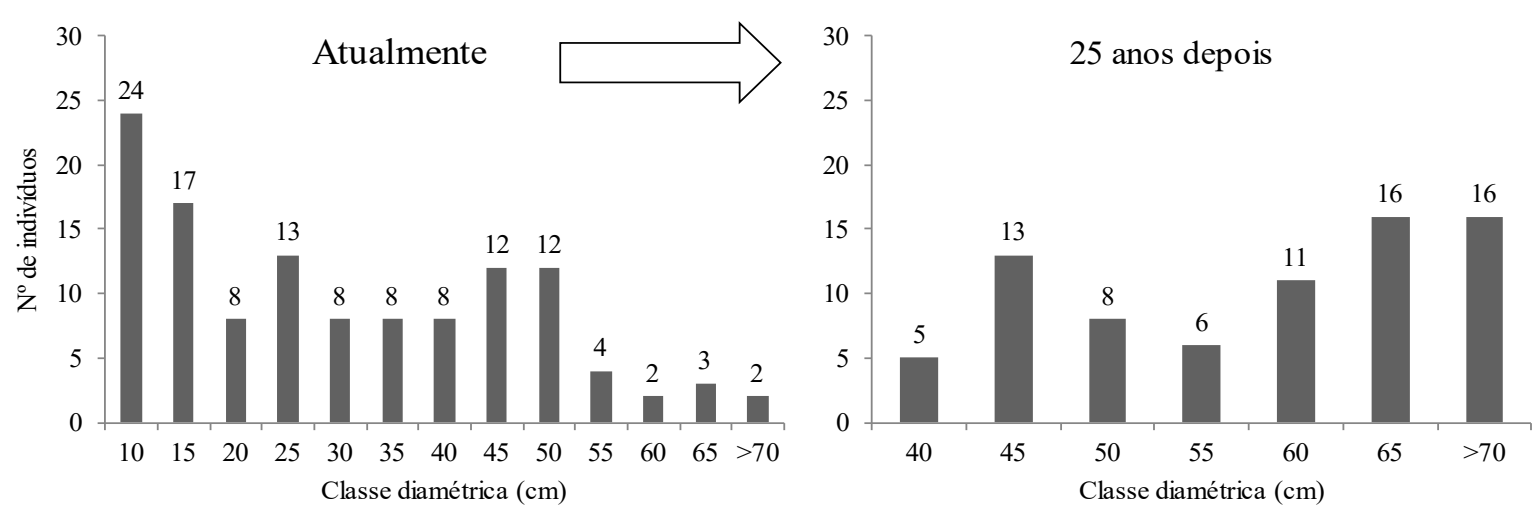

FIGURA 6: Distribuição diamétrica futura com base no incremento médio anual em diâmetro para as classes comerciais em Floresta de Araucária, Lages - SC.

FIGURE 6: Future diameter distribution based on the average annual increment in diameter for the commercial classes in Araucaria Forest, Lages, SC state.

Atualmente são 43 indivíduos acima de $40 \mathrm{~cm}$ de dap por hectare e no futuro serão 75, em se mantendo a taxa de incremento, o que representa um aumento de $74,42 \%$ nas classes comerciais, acima de $40 \mathrm{~cm}$ e com possibilidade de corte na floresta. Contudo, proporcionando um manejo adequado da área, a dinâmica de crescimento em estrutura diamétrica poderá aumentar o número de árvores das classes comerciais, pois como comprovado, as classes de menor diâmetro estão crescendo menos que a média, o que pode contribuir para a diminuição de árvores com maiores dimensões para corte no futuro. Essa informação é importante para o manejo, uma vez que sugere que o ciclo de corte de 25 anos, adotado no estudo, poderia ser diminuído com aumento na taxa de crescimento (LÓPEZ; VILLALBA; CLAROS, 2012).

Considerando a relação negativa com índices de competição (descrita acima) e o pequeno aumento em árvores acima de $40 \mathrm{~cm}$ para um ciclo de 25 anos, há necessidade de intervenções silviculturais, pois diminuirá a densidade de indivíduos, proporcionará melhores condições de recursos para o crescimento, abertura do dossel, aumento da regeneração natural, aumento das taxas de incremento, bem como fonte de renda, cumprindo as metas do manejo florestal.

\section{CONCLUSÕES}

O estudo demonstrou a possibilidade de manejo com retirada de madeira para um ciclo de corte, pois parte das árvores na floresta já atingiram seu potencial em crescimento. A taxa decrescente de incremento em diâmetro nas menores classes indicou que o não manejo ou somente a conservação, pode não ser a melhor maneira de perpetuidade em estrutura diamétrica da floresta e dinâmica de crescimento.

Correlação negativa entre incremento e índices de competição indica a necessidade de intervenções silviculturais com redução de densidade, já que atrasar o período de intervenções por um longo período de tempo prejudica a retomada do incremento.

Os resultados contribuem para a elaboração da gestão sustentável de Floresta com Araucária. 


\section{AGRADECIMENTOS}

Se expressa o agradecimento ao Conselho Nacional de Desenvolvimento Científico e Tecnológico, CNPq, pelo apoio financeiro para a realização do projeto, Chamada Universal 14/2013, pelo qual foi possível apresentar resultados da proposta, bem como ao proprietário do povoamento nativo de Araucária que permitiu a realização e coleta de dados para a concretização da pesquisa.

\section{REFERÊNCIAS}

ALVARES, C. A. et al. Köppen's climate classification map for Brazil. Meteorologische Zeitschrift, Swiss, v. 22, n. 6, p. 711-728, 2013.

ASSMAN, E. Waldertargskunde. Müchen: Bayr; Landw, 1961. 490 p.

ASSMANN, E. The principles of forest yield study. Oxford: Pergamon Press, 1970. 506 p.

BAUHUS, J.; PUETTMANN, K.; MESSIER, C. Silviculture for old-growth attributes. Forest Ecology and Management, Netherlands, v. 258, p. 525-537, jan. 2009.

BECKERT, S. M.; ROSOT, M. A. D.; ROSOT, N. C. Crescimento e dinâmica de Araucaria angustifolia (Bert.) O. Ktze. em fragmento de Floresta Ombrófila Mista. Scientia Forestalis, Piracicaba, v. 42, n. 102, p. 209-218, jun. 2014.

BURGER, H. Baumkrone und zuwachs in zwei hiebsreifen fichtenbeständen. Mitteilungen der Schweizerischen Anstalt für das Forstliche Versuchswesen, Wesen, v. 21, p. 147-176, 1939.

CATTANEO, N. et al. Sex-related, growth-climate association of Araucaria angustifolia in the neotropical ombrophilous woodlands of Argentina. Dendrochronologia, Italy, v. 31, p. 147-152, 2013. http://dx.doi.org/10.1016/j.dendro.2013.01.005

CARVALHO, P. E. R. Espécies Florestais Brasileiras: recomendações silviculturais, potencialidades e uso da madeira. In: EMBRAPA. Centro Nacional de Pesquisa de Florestas. Espécies Florestais Brasileiras. Colombo: Embrapa; CNPF, 1994, 640p.

CUNHA, T. A.; FINGER, C. A. G. Competição assimétrica e o incremento diamétrico de árvores individuais de Cedrela odorata L. na Amazônia ocidental. Acta Amazônica, Manaus, v. 43, n. 1, p. 9-18, 2013. DURLO, M. A.; DENARDI, L. Morfometria de Cabralea canjerana, em mata secundaria nativa do Rio Grande do Sul. Ciência Florestal, Santa Maria, v. 8, n. 1, p. 55-66, 1998.

EMPRESA DE PESQUISA AGROPECUÁRIA E EXTENSÃO RURAL DE SANTA CATARINA. Dados e informações biofísicas da Unidade de Planejamento Regional Planalto Sul Catarinense UPR 3. Florianópolis: EPAGRI; CIRAM, 2002. $70 \mathrm{p}$.

FOOD AND AGRICULTURE ORGANIZATION OF THE UNITED NATIONS. State of the World's Forest. [S.1.]: FAO, 2007. Disponível em: <http://www.fao.org/forestry/site/sofolen>. Acesso em: 25 mar. 2016.

GIRAUDO, A. R. et al. Biodiversity status of the interior Atlantic forest of Argentina. In: GALINDO-LEAL, C.; CÂMARA, V. (Ed.). The Atlantic Forest of South America: biodiversity status, threats, and outlook. Washington: Island Press, 2003. p. 160-180.

HESS, A. F. et al. Proposta de manejo de Araucaria angustifólia utilizando o quociente de Liocourt e análise de incremento, em propriedade rural no município de Lages, SC. Pesquisa Florestal Brasileira, Colombo, v. 30, n. 64, p. 337-345, nov./dez. 2010.

HESS, A. F.; SCHNEIDER, P. R.; FINGER, C. A. G. Crescimento em diâmetro de Araucaria angustifolia (Bertol.) Kuntze em função da idade, em três regiões do Rio Grande do Sul. Ciência Florestal, Santa Maria, v. 19, n. 1, p. 7-22, jan./mar. 2009.

HOSOKAWA, R. T.; SOLTER, F. Manejo Florestal. Curitiba: UFPR, 1995. 43 p.

HUECK, K. As florestas da América do Sul. Brasília: Editora da Universidade de Brasília, 1972.

LAURANCE, W. F. et al. Inferred longevity of Amazonian rainforest trees based on a long-term demographic study. Forest Ecology and Management, Netherlands, v. 190, p. 131-143, 2004.

LEITE, P. F.; KLEIN, R. M. Vegetação. In: IBGE. Diretoria de Geociências. Geografia do Brasil: Região Sul. Rio de Janeiro: IBGE, 1990. 420 p. 
LÓPEZ, L.; VILLALBA, R.; CLAROS, M. P. Ritmos de crecimiento diamétrico en los bosques secos tropicales: aportes al manejo sostenible de los bosques de la provincia biogeográfica del Cerrado Boliviano. Bosque, Chile, v. 33, n. 2, p. 211-219, 2012.

MATTOS, P. P. et al. Crescimento de Araucaria angustifolia na Reserva Florestal Embrapa/Epagri, Caçador, SC. Pesquisa Florestal Brasileira, Colombo, n. 55, p. 107-114, jul./dez. 2007.

MITSCHERLICH, G. Wald, Wachstum und Umwelt. 1. Bd.: Form und Wachstum von Baum und Bestand. 2. Auf. Frankfurt: Sauerländer's Verlag, 1978. 144 p.

ORELLANA, E.; KOEHLER, A. B. Relações morfométricas de Ocotea odorifera (Vell.) Rohwer. Revista Acadêmica Ciências Agrárias e Ambientais, Curitiba, v. 6, n. 2, p. 229-237, abr./jun. 2008.

PADOIN, V.; FINGER, C. A. G. F. Relações entre as dimensões da copa e a altura das árvores dominantes em povoamentos de Pinus taeda L. Ciência Florestal, Santa Maria, v. 20, n. 1, p. 95-105, jan./mar. 2010. PRETZSCH, H. Forest dynamics, growth and yield. From measurement to model. Berlin: Springer, 2009. $664 \mathrm{p}$.

PRETZSCH, H.; DIELER, J. The dependency of the size-growth relationship of Norway spruce (Picea abies [L.] Karst.) and European beech (Fagus sylvatica [L.]) in forest stands on long-term site conditions, drought events, and ozone stress. Trees, Berlin, v. 25, p. 355-369, nov. 2011. 10.1007/s00468-010-0510-1 PRODAN, M. Holzmeßlehre. Frankfurt: JD Sauerländer's Verlag, 1965. 644 p.

ROZENDAAL, D. M. A.; GAMBOA, C. C. S.; ZUIDEMA, P. A. Timber yield projections for tropical tree species: The influence of fast juvenile growth on timber volume recovery. Forest Ecology and Management, Netherlands, v. 259, p. 2292-2300, feb. 2010.

SANQUETTA, C. R. et al. Inventários florestais: planejamento e execução. 2. ed. Curitiba: Multi-Graphic, 2009. $316 \mathrm{p}$.

SANTOS, A. T. et al. Determinação da época de desbaste pela análise dendrocronológica e morfométrica de Ocotea porosa (Ness \& Mart.) Barroso em povoamento não manejado. Ciência Florestal, Santa Maria, v. 25 , n. 3, p. 699-709, jul./set. 2015.

SOUZA, A. L. et al. Dinâmica de crescimento em diâmetro de uma floresta primária sem interferência: uma análise pelo tempo de passagem entre classes diamétricas. Revista Árvore, Viçosa, MG, v. 17, n. 2, p. 2-16, 1993.

SPEER, J. Fundamentals of tree-ring research. Tucson: The University of Arizona Press, 2010. 333 p. SPIECKER, H. Tree rings and forest management in Europe. Dendrochronologia, Italy, v. 20, n. 1-2, p. 191-202, 2002.

SULLIVAN, T. P.; SULLIVAN, D. S. Acceleration of old-growth structural attributes in lodgepole pine forest: tree growth and stand structure 25 years after thinning. Forest Ecology and Management, Netherlands, v. 365, p. 96-106, jan. 2016.

TONINI, H.; ARCO-VERDE, M. Morfologia da copa para avaliar o espaço vital de quatro espécies nativas da Amazônia. Pesquisa Agropecuária Brasileira, Brasília, v. 40, n. 7, p. 633-638, jul. 2005.

VELOSO, H. P.; RANGEL FILHO, A. L. R.; LIMA, J. C. A. Classificação da vegetação brasileira adaptada a um sistema universal. Rio de Janeiro: IBGE, 1991. 128 p. 